\title{
Datos, información y conocimiento
}

\section{Héctor Jaime Dulcé Moreno PhD}

\section{Docente UFPS}

Con frecuencia se presenta una confusión entre los significados de los términos: dato, información y conocimiento. Para algunos autores estos términos, pertenecen exclusivamente al campo de la teoría de la comunicación, y son irrelevantes. Consideramos que, para toda persona interesada en la generación de conocimiento, se hace necesario no sólo tener claridad en los significados, sino también valorar la importancia de cada uno, por el hecho que son elementos esenciales, en los procesos educativos y de investigación.

Sin profundizar en teorías, se puede afirmar que la diferencia entre dato, información y conocimiento se da principalmente en el grado de procesamiento o sistematización de las interacciones del sujeto con el objeto de estudio.

En primera instancia, el dato o registro se podría considerar como una información pre-primaria, resultado de la interacción elemental entre el sujeto y el objeto. En la que el sujeto hace uso de las herramientas o instrumentos para su recolección o medida. La validez de dichos datos o registros depende tanto del instrumento como de la técnica utilizada.

La información primaria, resultado de un primer grado de procesamiento o sistematización de datos, está a asociada a la investigación científica, la cual puede ser considerada como uno de los posibles productos de investigación; ya que mediante artículos o libros se presentan los resultados del proceso investigativo. De acuerdo con la anterior consideración, un artículo científico debe incluir elementos tales como: los antecedentes, la justificación, los propósitos u objetivos, los instrumentos, las técnicas, los registros, el procesamiento de registros, y análisis de resultados con sus correspondientes conclusiones. Adicionalmente se presentan las referencias, que constituyen el marco conceptual que sustenta uno o varios de los elementos de la investigación.

Por otra parte, la información secundaria, considerada como un segundo grado de procesamiento, se obtiene de la sistematización de la información primaría. Un ejemplo de ello son los artículos de revisión, donde lo más importante es contar con un suficiente número de referencias que garanticen la representatividad del universo de la temática tratada.

La información por sí sola no es conocimiento, ya que el conocimiento se elabora por la interacción del sujeto con las diversas fuentes de información, en los diferentes grados de procesamiento o presentación, pero con el referente propio de un individuo o de una comunidad. Hoy en día, con el desarrollo de alternativas de acceso a la información, como el internet, es posible acceder a la información en diferentes formas de presentación; opción importante y necesaria especialmente en los procesos de aprendizaje.

Adicionalmente, las bases de información garantizan otro grado de sistematización que facilita la búsqueda de una información específica mediante el uso de palabras claves, nombres de autores, áreas de conocimiento, entre otras, que facilitan la visualización de las fuentes de información en diferentes grados de procesamiento.

En la actualidad se está presentando un fenómeno, que se podría denominar comercialización y hasta usura de la información o del conocimiento. Debido al cobro de tarifas, que no están al alcance del común de la gente, se limita el acceso a la información básica. Por ejemplo, el costo de un artículo científico, en algunas bases de datos, supera el valor de los treinta dólares, cercano a la quinta parte de un salario mínimo legal vigente en Colombia.

La Revista Respuestas, de acuerdo con su propósito fundamental, busca contribuir con la difusión del conocimiento científico mediante la publicación de resultados de investigación en las áreas de las Ciencias Naturales, las Ingenierías y Tecnologías, las Ciencias Médicas y de la Salud, las Ciencias Agrícolas, las Ciencias Sociales y Humanidades.

Para aumentar su visibilidad la revista busca cada día estar mejor Indexada y adecuadamente indizada. 